\title{
Tensões de água no solo no cultivo de mamoneira ${ }^{1}$
}

\author{
Gervásio Fernando Alves Rios ${ }^{2}$, Luiz Gonsaga de Carvalho ${ }^{3}$, \\ Bruno Montoani Silva ${ }^{4}$, Fátima Conceição Rezende 3 , Geraldo Magela Pereira ${ }^{3}$
}

\begin{abstract}
Soil water tensions on castor bean crop

With the increasing global demand for energy, more attention has been directed to the production of oilseed plants as alternative energy sources, being irrigation one of the technologies used to increase yield. This research aimed to evaluate the effect of different soil water tensions and respective moisture depletions on castor bean (AL Guarany 2002 cultivar). The experimental design was randomized blocks, with five soil water tensions ( $15 \mathrm{kPa}, 30 \mathrm{kPa}, 45 \mathrm{kPa}, 60 \mathrm{kPa}$ and $75 \mathrm{kPa})$ and four replications. The water use efficiency (WUE) and yield were evaluated according to the soil water tension and respective moisture depletion. The soil coverage factor and plant height were also evaluated during the crop cycle, being respectively observed increases up to 125 and 200 days after transplanting. The WUE, following the order of racemes produced - primary $\left(W_{U} E_{p}\right)$, secondary $\left(W_{U} E_{S}\right)$, tertiary $\left(W_{U} E_{T}\right)$ and total for the crop $\left(\mathrm{WUE}_{\mathrm{C}}\right.$ ) - decreased linearly with the increasing water stress, being the highest values observed for the treatment with the lowest tension $(15 \mathrm{kPa})$. For all irrigation treatments, the highest WUE and yield were observed in secondary racemes. The highest yield was $1,994.14 \mathrm{~kg} \mathrm{ha}^{-1}$, for the tension of $15 \mathrm{kPa}$ and soil moisture depletion of $28 \%$, while the lowest one was $773.91 \mathrm{~kg} \mathrm{ha}^{-1}$, for the tension of $75 \mathrm{kPa}$ and soil moisture depletion of $83 \%$.
\end{abstract}

KEY-WORDS: Ricinus communis L.; irrigation management; soil coverage factor; soil moisture depletion.

\section{INTRODUÇÃO}

A mamoneira (Ricinus communis L.) é uma cultura com importância econômica crescente, principalmente com o advento, na última década, do Programa Nacional de Produção e Uso do Biodiesel (PNPB). De acordo com as estimativas da Conab (2014), em junho de 2014, a área cultivada

\section{RESUMO}

Com a crescente demanda energética mundial, maior atenção tem sido destinada à produção de oleaginosas como fontes alternativas de energia, sendo a irrigação uma das tecnologias adotadas para o aumento da produção. Objetivouse, com este trabalho, estudar o efeito de diferentes tensões e respectivos esgotamentos de água no solo, no cultivo da mamoneira (cv. AL Guarany 2002). Foi utilizado o delineamento de blocos ao acaso, com cinco tensões de água no solo (15 kPa, $30 \mathrm{kPa}, 45 \mathrm{kPa}, 60 \mathrm{kPa}$ e $75 \mathrm{kPa}$ ) e quatro repetições. Foi avaliada a eficiência de uso da água (EUA) e a produtividade, em função da tensão de água no solo e respectiva fração de esgotamento de água. Além disso, avaliou-se o fator de cobertura do solo e altura das plantas, durante o ciclo da cultura, verificando-se o seu aumento até 125 e 200 dias após o transplantio, respectivamente. A EUA, seguindo a ordem dos racemos produzidos - primário $\left(\mathrm{EUA}_{\mathrm{P}}\right)$, secundário $\left(\mathrm{EUA}_{\mathrm{S}}\right)$, terciário $\left(\mathrm{EUA}_{\mathrm{T}}\right)$ e total da cultura $\left(\right.$ EUA $_{C}$ ) - foi linearmente reduzida com o aumento da restrição hídrica, sendo os maiores valores observados para o tratamento à menor tensão $(15 \mathrm{kPa})$. Para todos os tratamentos de irrigação, a maior EUA e produtividade foram observadas nos racemos secundários. A maior produtividade foi de $1.994,14 \mathrm{~kg} \mathrm{ha}^{-1}$, para a tensão de $15 \mathrm{kPa}$ e fração de esgotamento de água no solo de $28 \%$, e a menor de $773,91 \mathrm{~kg} \mathrm{ha}^{-1}$, para a tensão de $75 \mathrm{kPa}$ e fração de esgotamento de água no solo de $83 \%$.

PALAVRAS-CHAVE: Ricinus communis L.; manejo de irrigação; fator de cobertura do solo; depleção de água no solo.

com mamoneira, na safra 2013/2014, foi de 102,2 mil hectares. Nessa safra, os Estados da Bahia e Ceará abrangeram $92,1 \%$ da área total nacional cultivada, correspondendo a 81,8 e 12,3 mil hectares, contribuindo, também, para as maiores produções (67,2 e 5,2 mil toneladas, respectivamente), sendo a produção nacional de 76,3 mil toneladas. A melhor média de produtividade foi prevista para o Estado

1. Trabalho recebido em ago./2013 e aceito para publicação em jul./2014 ( $\mathrm{n}^{\circ}$ registro: PAT 26235).

2. Universidade de Brasília (UnB), Faculdade de Agronomia e Medicina Veterinária, Engenharia Agrícola e Agronômica de Água, Solo e Planta, Brasília, DF, Brasil.E-mail: gervasiorios@yahoo.com.br.

3. Universidade Federal de Lavras (UFLa), Departamento de Engenharia, Lavras, MG, Brasil.E-mails: lgonsaga@deg.ufla.br, frezende@deg.ufla.br, geraldop@deg.ufla.br.

4. Universidade Federal de Lavras (UFLa), Departamento de Ciência do Solo, Lavras, MG, Brasil. E-mail: brunoms3@yahoo.com.br. 
da Bahia, com $822 \mathrm{~kg} \mathrm{ha}^{-1}$, frente à média nacional de $747 \mathrm{~kg} \mathrm{ha}^{-1}$.

O principal produto de interesse econômico resultante da mamoneira é o óleo de rícino, que possui inúmeras aplicações na indústria química de fabricação de tintas, vernizes, cosméticos, fluidos hidráulicos e plásticos (Costa 2010). Além dessas aplicações, tem sido utilizado como aditivo ao diesel, para a produção de biodiesel (Sausen \& Rosa 2010).

Ao longo do ciclo da mamoneira, são produzidas várias ordens de racemos, sendo o primário, secundário e terciário os mais importantes. Seu enchimento depende das condições ambientais, época de cultivo e características de cada cultivar, variando a contribuição de cada fator na produtividade total (Vijaya Kumar et al. 1997, Koutroubas et al. 1999, Veríssimo et al. 2009).

Os racemos primários foram os que mais contribuíram para a produtividade total, de acordo com Vijaya Kumar et al. (1997), os quais desenvolveram experimento na Índia, com a cultivar Aruna, em espaçamento $0,25 \mathrm{~m} \times 0,60 \mathrm{~m}$, relacionando a ordem de racemos e produtividade. Contudo, Savy Filho et al. (1990) e Souza et al. (2007a, 2007b), trabalhando com as cultivares IAC 226 e BRS Nordestina, respectivamente, afirmaram que os racemos secundários são os que representam o maior percentual da produtividade.

No entanto, a mamoneira perde grande parte da sua produção econômica, em regiões cuja precipitação pluvial acumulada no período chuvoso seja inferior a $500 \mathrm{~mm}$ (Barros Júnior et al. 2008). Destaca-se, ainda, que, em regiões com estação seca definida, ou mesmo com a presença de veranicos durante o ciclo, o desenvolvimento dos frutos e a produção podem ser prejudicados. De acordo com Freitas et al. (2010), para crescer, se desenvolver e produzir satisfatoriamente, a mamoneira necessita de suprimento hídrico diferenciado, nas suas fases fenológicas, e, portanto, requer manejo compatível com a sua capacidade de retirada de água do solo.

Souza et al. (2007a, 2007b) relacionaram quatro épocas de plantio da cultivar BRS Nordestina, a partir de dezembro - equidistantes em 30 dias, com duas épocas de irrigação suplementar (antes e depois do período chuvoso). Esses autores verificaram que a produção de grãos foi positivamente correlacionada com o número de racemos por planta e que as sementes dos racemos secundários e terciários possuíam maior teor de óleo e eram mais pesadas que as do primário.
Resultados obtidos por Biscaro et al. (2012) indicaram que a irrigação localizada influenciou significativamente na produção de duas cultivares de mamona, obtendo-se $1.513,9 \mathrm{~kg}$ e $1.452,4 \mathrm{~kg}$ de sementes ha ${ }^{-1}$, para as cultivares IAC 80 e IAC 2028, respectivamente. Essas cultivares foram irrigadas com lâminas equivalentes a $0 \%, 25 \%, 50 \%, 100 \%$ e $150 \%$ da evapotranspiração da cultura e, de acordo com os autores, a quantidade de água aplicada, mesmo de forma suplementar, foi importante para aumentar sua produtividade.

A eficiência de uso da água (EUA) relaciona a produção de biomassa, em razão da quantidade de água aplicada. Elevar os níveis de EUA pode ser bastante complexo, porém, um dos meios adotados para elevar esse parâmetro é realizando-se o manejo adequado da irrigação.

Barros Júnior et al. (2008) relataram que a EUA aumentou com o volume de água aplicado na irrigação das cultivares Nordestina e Paraguaçu, cultivadas em vasos e em ambiente protegido, ou seja, as plantas apresentaram maior capacidade de reverter o volume de água consumido em produção de matéria seca, sempre que o conteúdo de água no solo foi aumentado. De acordo com esses autores, essas cultivares possuem alto potencial produtivo, por atingirem EUA de $2,83 \mathrm{~kg} \mathrm{~m}^{-3} \mathrm{e} 2,78 \mathrm{~kg} \mathrm{~m}^{-3}$, respectivamente para as cultivares Nordestina e Paraguaçu, quando submetidas a $100 \%$ de água disponível no solo, sendo sensíveis em resposta à água.

Situação similar foi encontrada por Lacerda et al. (2009), com a cultivar Paraguaçu, cultivada em vasos, em ambiente protegido, sob quatro níveis de água disponível no solo $(70 \%, 80 \%, 90 \%$ e $100 \%)$, em que a EUA foi maior quando as plantas foram conduzidas no nível mais alto de disponibilidade de água no solo.

Já Duarte et al. (2012), trabalhando com girassol irrigado por gotejamento, com três períodos de suspensão de irrigação e sem suspensão de irrigação, verificaram que a EUA, na produção de óleo e de aquênios, foi maior no tratamento irrigado com a menor lâmina. A diferença entre os resultados obtidos para a cultura da mamona e do girassol pode ser devido às condições de cultivo, ou mesmo das características das culturas.

Este trabalho foi conduzido com o objetivo de avaliar a EUA, em função da tensão de água e respectiva fração de esgotamento da água no solo, na produtividade (por ordem de racemos e total) da cultura da mamoneira irrigada por gotejamento. 


\section{MATERIAL E MÉTODOS}

O experimento foi conduzido de 24/01/2008 a 30/08/2008 (220 dias), no Departamento de Engenharia da Universidade Federal de Lavras (UFLa), em Lavras (MG) $\left(21^{\circ} 14^{\prime} \mathrm{S}, 45^{\circ} 00^{\prime} \mathrm{W}\right.$ e altitude de $\left.918,8 \mathrm{~m}\right)$. O clima da região é classificado como "Cwa", segundo Köppen, com valores médios anuais de temperatura de $19,4^{\circ} \mathrm{C}$, umidade relativa de $76,2 \%$ e precipitação pluvial total anual de $1.530 \mathrm{~mm}$ (Dantas et al. 2007).

O solo foi classificado como Latossolo Vermelho distroférrico (Embrapa 2006), com textura argilosa. A curva característica de água do solo foi determinada no Laboratório de Relação Água-Solo-Planta do Departamento de Engenharia da UFLa. Foram coletadas amostras, nas camadas de 0-0,20 m e 0,20-0,40 $\mathrm{m}$ de profundidade, e, com os dados de umidade associada às suas respectivas tensões, obteve-se o ajuste da curva de retenção de água no solo, com o auxílio do programa SWRC, desenvolvido por Dourado Neto et al. (2000), que descreve o comportamento da umidade do solo, em função da tensão nas camadas de $0-0,20 \mathrm{~m}$ e 0,20-0,40 $\mathrm{m}$ de profundidade, conforme as Equações 1 e 2 , respectivamente:

$$
\begin{aligned}
& \theta=0,221+\frac{(0,598-0,221)}{\left[1+\left(0,288 \cdot\left|\psi_{\mathrm{m}}\right|\right)^{1,879}\right]^{0,468}} \quad \mathrm{R}^{2}=0,962 \\
& \theta=0,230+\frac{(0,604-0,230)}{\left[1+\left(0,265 \cdot\left|\psi_{\mathrm{m}}\right|\right)^{2,024}\right]^{0,506}} \quad \mathrm{R}^{2}=0,957
\end{aligned}
$$

em que $\theta$ é a umidade do solo $\left(\mathrm{cm}^{3} \mathrm{~cm}^{-3}\right)$ e $\Psi m$ o potencial matricial da água no solo $(\mathrm{kPa})$.

A umidade do solo, na capacidade de campo $(\theta \mathrm{cc})$, foi considerada à tensão de $10 \mathrm{kPa}$, correspondente às umidades de $0,361 \mathrm{~cm}^{3} \mathrm{~cm}^{-3}$, na camada de $0-0,20 \mathrm{~m}$, e de $0,359 \mathrm{~cm}^{3} \mathrm{~cm}^{-3}$, na camada de 0,20-0,40 m.

O preparo do solo consistiu de procedimentos convencionais de subsolagem, aração e gradagem. O experimento foi implantado por mudas da cultura de mamona variedade AL Guarany 2002, previamente formadas em viveiro. No transplantio das mudas, efetuou-se uma adubação, na dosagem de $0,05 \mathrm{~kg}$ cova $^{-1}$ do formulado NPK 8-28-16, com base na análise química do solo. Aos 30 dias após o plantio (DAP), realizou-se a adubação de cobertura, com $0,045 \mathrm{~kg}$ de sulfato de amônio por planta.

Os tratamentos (T1 a T5) consistiram na reposição de água ao solo, quando as tensões da água no solo atingissem, respectivamente, $15 \mathrm{kPa}, 30 \mathrm{kPa}$,
$45 \mathrm{kPa}, 60 \mathrm{kPa}$ e $75 \mathrm{kPa}$, a 0,20 m de profundidade. Para esses tratamentos, as respectivas frações de esgotamento da água do solo foram calculadas com base nas Equações 3, 4 e 5:

$$
\begin{aligned}
& \mathrm{f}=(\mathrm{CRA} / \mathrm{CTA}) \cdot 100 \\
& \mathrm{CRA}=(\theta \mathrm{cc}-\theta \mathrm{a}) \cdot \mathrm{Z} \\
& \mathrm{CTA}=(\theta \mathrm{cc}-\theta \mathrm{pm}) \cdot \mathrm{Z}
\end{aligned}
$$

em que $f$ é a fração de esgotamento de água no solo (\%), CRA a capacidade real de água no solo (decimal), CTA a capacidade total de água no solo (decimal), $\theta a$ a umidade do solo na tensão definida para cada tratamento $\left(\mathrm{cm} \mathrm{cm}^{-3}\right), \theta p m$ a umidade do solo no ponto de murcha permanente $\left(\mathrm{cm} \mathrm{cm}^{-3}\right)$ e $Z$ a profundidade efetiva do sistema radicular $(\mathrm{mm})$.

Cada parcela experimental possuía área de $54,0 \mathrm{~m}^{2}$, com três linhas e espaçamento entre si de $2,4 \mathrm{~m}$, contendo dez plantas por linha, espaçadas em $0,75 \mathrm{~m}$, das quais somente as seis plantas centrais constituíram a parcela útil.

O sistema de irrigação utilizado foi o de gotejamento, com gotejadores autocompensantes espaçados em $0,75 \mathrm{~m}$, operando à vazão e pressão nominal de $2,2 \mathrm{~L} \mathrm{~h}^{-1}$ e $200 \mathrm{kPa}$, respectivamente, formando uma faixa molhada na superfície do solo (fw) de 33,3\%, mantida praticamente constante. O momento de irrigação foi determinado instalando-se tensiômetros às profundidades de $0,20 \mathrm{~m}$ e $0,40 \mathrm{~m}$, em cada tratamento. As leituras dos tensiômetros foram realizadas diariamente entre $6 \mathrm{~h}$ e $8 \mathrm{~h}$ da manhã, utilizando-se um leitor digital de punção (tensímetro). As irrigações foram realizadas toda vez que as leituras da tensão matricial, à profundidade de $0,20 \mathrm{~m}$, atingissem os valores críticos pré-estabelecidos para cada tratamento, e o tempo de irrigação foi calculado com base na Equação 6:

$$
\mathrm{t}=\frac{\frac{(\theta c c-\theta a) \cdot Z \cdot f w}{\text { Ea.CUC }} \cdot A p}{e \cdot q}
$$

em que $t$ é o tempo de irrigação (h), $E a$ a eficiência de aplicação de água do sistema de irrigação $(0,90)$, CUC o coeficiente de uniformidade do sistema de irrigação $(0,95), A p$ a área útil ocupada por planta $\left(\mathrm{m}^{2}\right)$, e o número de gotejadores por planta e $q$ a vazão média dos gotejadores $\left(\mathrm{L} \mathrm{h}^{-1}\right)$.

A precipitação efetiva retida à profundidade efetiva do sistema radicular considerado foi calculada conforme a Equação 7: 
$\mathrm{Pe}_{(\mathrm{i})}= \begin{cases}\mathrm{Pt}_{(\mathrm{i})} & \mathrm{se} \mathrm{Pt}_{(\mathrm{i})} \leq\left(\mathrm{CRA}_{(\mathrm{i})} \cdot \mathrm{fw}\right) \\ \mathrm{CRA}_{(\mathrm{i})} & \mathrm{se} \mathrm{Pt}_{(\mathrm{i})} \geq\left(\mathrm{CRA}_{(\mathrm{i})} \cdot \mathrm{fw}\right)\end{cases}$

em que $P e$ é a precipitação efetiva $(\mathrm{mm}), P t$ a precipitação total (mm) e a $C R A$ calculada conforme a Equação 4.

Para o critério de definição das fases fenológicas, adotou-se a fração de cobertura do solo pelo dossel da cultura, calculada conforme a Equação 8:

$f c=\left\{\begin{array}{lll}\frac{\pi \cdot \mathrm{Rcob}^{2}}{\mathrm{Sp} \cdot \mathrm{Sf}} \cdot 100 & \text { se } & \text { Rcob } \leq \frac{\mathrm{Sp}}{2} \\ \frac{2 \cdot \mathrm{Rcob}}{\mathrm{Sf}} \cdot 100 & \text { se } & \text { Rcob }>\frac{\mathrm{Sp}}{2}\end{array}\right.$

em que $f c$ é a fração de cobertura do solo (\%), Rcob o raio de cobertura do dossel da cultura (m), $S f$ o espaçamento entre fileiras de plantio (m) e $S p$ o espaçamento entre plantas na fileira de plantio (m).

As fases fenológicas foram determinadas no tratamento T1 e definidas pelos seguintes critérios: Fase I - dias após o plantio (DAP), referente ao crescimento vegetativo até quando a $f c$ atingisse $10 \%$, conforme Allen et al. (1998); Fase II - do término da fase I até o DAP, em que mais de $50 \%$ das plantas atingiram o primeiro racemo; Fase III - fase intermediária (floração e/ou frutificação), desde o término da fase II até o DAP, em que mais de $50 \%$ dos frutos dos racemos terciários atingiram a maturação fisiológica; Fase IV - fase final da maturação dos frutos, desde o término da fase III até o final do ciclo produtivo.

A altura da planta, definida a partir da superfície do solo até o meristema apical (gema) mais alto, foi avaliada mensalmente, utilizando-se uma régua. Ao final do ciclo produtivo, quando $2 / 3$ dos frutos atingiram a maturação, avaliou-se a fração de cobertura do solo e a EUA na produção de grãos, pela relação entre a produtividade de grãos a $10 \%$ em base úmida e a lâmina de irrigação aplicada em cada tratamento (LI). A produtividade, em função da fração de esgotamento da água do solo, também foi avaliada. A EUA e a produtividade foram calculadas para cada ordem de racemo: primário $\left(\mathrm{EUA}_{\mathrm{p}}\right)$, secundário $\left(\mathrm{EUA}_{\mathrm{S}}\right)$, terciário $\left(\mathrm{EUA}_{\mathrm{T}}\right)$ e total da cultura $\left(\mathrm{EUA}_{\mathrm{C}}\right)$.

$\mathrm{O}$ delineamento estatístico adotado foi o de blocos ao acaso, com cinco tratamentos e quatro repetições. As variáveis foram submetidas à análise de variância e regressão, selecionando-se os modelos de regressão que apresentaram maiores níveis de significância do coeficiente de determinação $\left(\mathrm{R}^{2}\right)$ e de seus coeficientes de ajuste. As análises estatísticas foram feitas com o auxílio do programa estatístico Sisvar (Ferreira 2011).

\section{RESULTADOS E DISCUSSÃO}

Na Tabela 1, estão relacionados os dados relativos à irrigação, durante o período de condução do experimento. Os respectivos fatores de esgotamento de água no solo (f) variaram de $28,1 \%$ a $83,0 \%$, para os tratamentos T1 a T5, e a lâmina total de água aplicada variou de $628,6 \mathrm{~mm}$ a $502,8 \mathrm{~mm}$, considerando-se irrigações e precipitação efetiva. A precipitação total, durante o experimento, foi de $633,6 \mathrm{~mm}$, sendo que $95 \%$ desse total foram registrados até os 95 dias após o plantio (DAP). A lâmina de água aplicada em todos os tratamentos, até os $95 \mathrm{DAP}$, foi de $62,4 \mathrm{~mm}$ e, a partir dessa data, iniciaram-se os tratamentos de irrigação, coincidindo com o surgimento das primeiras flores. Verificou-se que o turno de rega aumentou com o aumento das tensões de água no solo.

Complementando o presente estudo, avaliou-se o crescimento vegetativo das plantas pela fração de cobertura do solo e respectiva altura (Figura 1), distribuído nas quatro fases fenológicas. Portanto, adotou-se o tratamento T1 (15 kPa), considerando-se

Tabela 1. Resumo dos dados referentes à irrigação, na cultura da mamoneira (Lavras, MG, 2008).

\begin{tabular}{|c|c|c|c|c|c|c|c|c|c|c|c|}
\hline \multirow{2}{*}{ Tratamento } & \multirow{2}{*}{$f(\%)$} & \multicolumn{2}{|c|}{ Tensão $(\mathrm{kPa})$} & \multicolumn{7}{|c|}{ Lâmina $(\mathrm{mm})$} & \multirow{2}{*}{$\begin{array}{c}\text { TR } \\
\text { (dias) }\end{array}$} \\
\hline & & Estab. & Média & LIinic & LIpós & $\mathrm{Pt}$ & $\mathrm{Pe}$ & LIpós + Pe & LIm & LItot & \\
\hline 15 & 28,1 & 15 & $14 \pm 1^{*}$ & 62,4 & 452,1 & 633,6 & 176,4 & 628,6 & 6,1 & 514,5 & $2 \pm 0,3^{*}$ \\
\hline 30 & 60,7 & 30 & $30 \pm 2 *$ & 62,4 & 445,7 & 633,6 & 176,4 & 622,1 & 10,9 & 508,1 & $5 \pm 1 *$ \\
\hline 45 & 72,7 & 45 & $44 \pm 5^{*}$ & 62,4 & 419,5 & 633,6 & 176,4 & 596,0 & 12,3 & 481,9 & $7 \pm 1^{*}$ \\
\hline 60 & 79,1 & 60 & $64 \pm 3^{*}$ & 62,4 & 393,2 & 633,6 & 176,4 & 569,6 & 12,7 & 455,6 & $9 \pm 1,5^{*}$ \\
\hline 75 & 83,0 & 75 & $73 \pm 2 *$ & 62,4 & 326,4 & 633,6 & 176,4 & 502,8 & 11,7 & 388,8 & $13 \pm 4^{*}$ \\
\hline
\end{tabular}

* Variação correspondente ao intervalo de confiança, a 5\%, pelo Teste t. f - fator de esgotamento de água no solo; Estab. e Média - tensão estabelecida e média, respectivamente, da água no solo, observada à profundidade de $0,2 \mathrm{~m}$; LIinic e LIpós - lâmina d'água aplicada antes e após diferenciação dos tratamentos, respectivamente; Pt e Pe - precipitação pluvial total e efetiva, respectivamente; LIm - lâmina d'água média aplicada (LIpós/74); LItot - lâmina total (LIinic + LIpós); TR - turno de rega. 


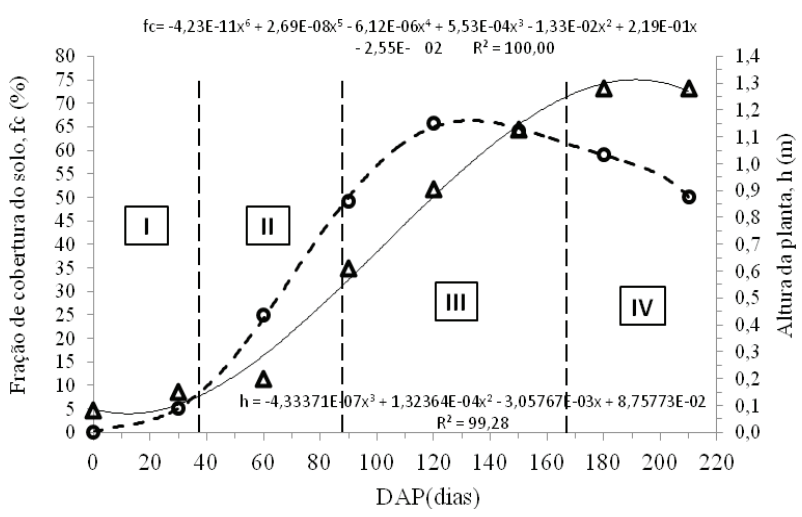

Figura 1. Fração de cobertura do solo (o) e altura da planta $(\Delta)$ do tratamento mantido próximo à capacidade de campo, durante o ciclo de cultivo, em dias após o plantio (DAP) (Lavras, MG, 2008).

esse como referência, em razão da menor limitação hídrica. Durante praticamente todo o ciclo, houve incremento na altura das plantas, cessando próximo aos 200 DAP, no estádio de maturação dos frutos ou senescência. Como a água é fator importante no crescimento e desenvolvimento vegetal, pode-se inferir que manter a água no solo próximo à capacidade de campo, para o solo em questão $(10 \mathrm{kPa})$, é uma forma de contribuir para o crescimento vegetal ideal para a mamoneira.

Ao longo das fases fenológicas I e II e até os 125 DAP da fase III, no tratamento T1, houve aumento da fração de cobertura do solo pelas plantas. Em sequência, ocorreu queda, até alcançar a última fase, sugerindo que, a partir da fase III, o crescimento vegetativo deixa de ser priorizado. A altura máxima atingida pela cultura foi de $1,3 \mathrm{~m}$, na fase IV, e a fração máxima de cobertura do solo equivaleu a $65 \%$, durante a fase intermediária III.

Lacerda et al. (2009) observaram que, aos 120 DAP, houve comportamento linear significativo entre área foliar e água disponível no solo, o que contribui para explicar o crescimento com tendência linear observado até os 120 DAP, na fase III (Figura 1). Silva et al. (2009) verificaram que a mamoneira cv. BRS Energia atingiu máximas respostas para altura de planta $(105,74 \mathrm{~cm})$, área foliar $\left(3.786,24 \mathrm{~cm}^{2}\right)$ e produtividade $\left(1.937,40 \mathrm{~kg} \mathrm{ha}^{-1}\right)$, quando irrigada com lâmina de $479,75 \mathrm{~mm}$, valor inferior ao que foi aplicado no tratamento T1, que foi de $690,9 \mathrm{~mm}$ (LIinic + LIpos + Pe).

Pôde-se verificar que a fração de cobertura do solo apresentou tendência semelhante à variação da altura da planta nas fases I, II e III. Na fase IV, a fração de cobertura se reduziu, devido à queda de folhas causada pela senescência.

Rodrigues et al. (2009), trabalhando com duas cultivares de mamona irrigadas com cinco níveis de reposição de água, avaliaram o número de folhas aos $48,90,132$ e 174 dias após a semeadura (DAS) e observaram que, após 100 DAS, ocorreu redução de folhas em todos os tratamentos, devido à senescência.

A eficiência do uso de água pela cultura $\left(E_{C} A_{C}\right)$ e por ordem de racemos primário $\left(E A_{p}\right)$, secundário $\left(\mathrm{EUA}_{\mathrm{S}}\right)$ e terciário $\left(\mathrm{EUA}_{\mathrm{T}}\right)$ é visualizada na Figura 2. Nota-se que, em ambas, a EUA tem tendência linear decrescente, à medida que se aumenta a tensão de água no solo. As maiores $\mathrm{EUA}_{\mathrm{P}}, \mathrm{EUA}_{\mathrm{S}}$, EUA $_{\mathrm{T}}$ e $\mathrm{EUA}_{\mathrm{C}}$ ocorreram quando o manejo de água foi mantido com a menor tensão $(15 \mathrm{kPa})$, correspondendo aos valores de $1,50 \mathrm{~kg} \mathrm{ha}^{-1} \mathrm{~mm}^{-1} ; 1,97 \mathrm{~kg} \mathrm{ha}^{-1} \mathrm{~mm}^{-1}$; $0,94 \mathrm{~kg} \mathrm{ha}^{-1} \mathrm{~mm}^{-1}$; e $4,41 \mathrm{~kg} \mathrm{ha}^{-1} \mathrm{~mm}^{-1}$, respectivamente. Esses resultados mostram que, nas condições avaliadas, a EUA é maior quando as irrigações são feitas em intervalos mais curtos.

Para a cultura do amendoim, Silva \& Beltrão (2000) verificaram que a eficiência no uso da água foi maior quando as plantas foram conduzidas no nível mais alto de disponibilidade de água no solo. Evidenciou-se que a cultura sob umidade no solo próxima ou na capacidade de campo atinge melhor eficiência nas suas funções metabólicas e fisiológicas, propiciando o aumento da EUA.

As produtividades de grãos, por ordem dos racemos secundário $\left(\mathrm{PR}_{\mathrm{S}}\right)$, terciário $\left(\mathrm{PR}_{\mathrm{T}}\right)$ e total da cultura $\left(\mathrm{PT}_{\mathrm{C}}\right)$, apresentaram comportamento qua-

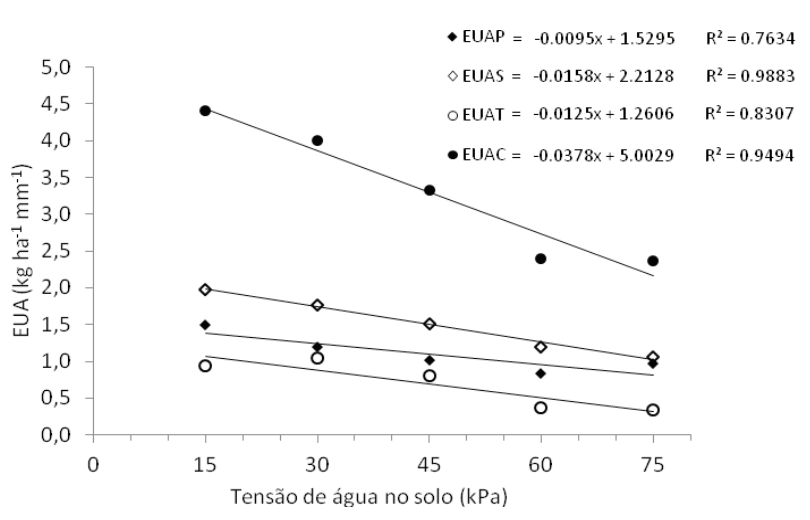

Figura 2. Eficiência de uso da água na produtividade da mamoneira $\left(\mathrm{EUA}_{\mathrm{C}}\right)$ e dos racemos primário $\left(\mathrm{EUA}_{\mathrm{p}}\right)$, secundário $\left(\mathrm{EUA}_{\mathrm{S}}\right)$ e terciário $\left(\mathrm{EUA}_{\mathrm{T}}\right)$, em função da tensão de água no solo (Lavras, MG, 2008). 
drático, em relação ao fator de esgotamento de água disponível no solo (f), correspondendo às tensões dos tratamentos T1 a T5. Percebeu-se que as maiores produtividades tendem a ocorrer nos tratamentos com menor limitação hídrica e observou-se redução na taxa média de produtividade, a partir do tratamento de $30 \mathrm{kPa}$ (T2), cujo valor de $f$ foi de $61 \%$ (Figura 3). Exceção ocorreu para a produtividade de racemos primários $\left(\mathrm{PR}_{\mathrm{P}}\right)$, que, por serem os primeiros a se desenvolverem, certamente, foram beneficiados pelo período chuvoso, no início do cultivo.

A $\mathrm{PR}_{\mathrm{P}}$ ajustou-se linearmente, com maior taxa de redução (cerca de $-6,68 \mathrm{~kg} \mathrm{ha}^{-1}$ por unidade porcentual de $f$ ), à semelhança do observado para o efeito da tensão matricial. As produtividades máximas da cultura, $\mathrm{PR}_{\mathrm{P}}, \mathrm{PR}_{\mathrm{S}}$ e $\mathrm{PR}_{\mathrm{T}}$ de $678,02 \mathrm{~kg} \mathrm{ha}^{-1}$, $891,44 \mathrm{~kg} \mathrm{ha}^{-1}$ e 424,68 $\mathrm{kg} \mathrm{ha}^{-1}$, respectivamente, foram obtidas no tratamento $\mathrm{T} 1(15 \mathrm{kPa})$.

A produtividade do racemo secundário foi $31,5 \%$ e $109,9 \%$ superior à produtividade observada nos racemos primários e terciários, respectivamente. Esses resultados foram semelhantes aos obtidos por Freitas et al. (2010) e Souza et al. (2007b), observando que, com o aumento da disponibilidade hídrica no solo, os racemos secundários foram os que mais contribuíram na produtividade total.

Destaca-se, ainda, que, para as condições climáticas da região, estação de cultivo e irrigação, onde foi desenvolvido este trabalho, a maior produtividade foi de $1.994,14 \mathrm{~kg} \mathrm{ha}^{-1}$, no tratamento T1 $(15 \mathrm{kPa})$, e a menor de $773,91 \mathrm{~kg} \mathrm{ha}^{-1}$, no tratamento T5 $(75 \mathrm{kPa})$. Com base na função obtida para produtividade da

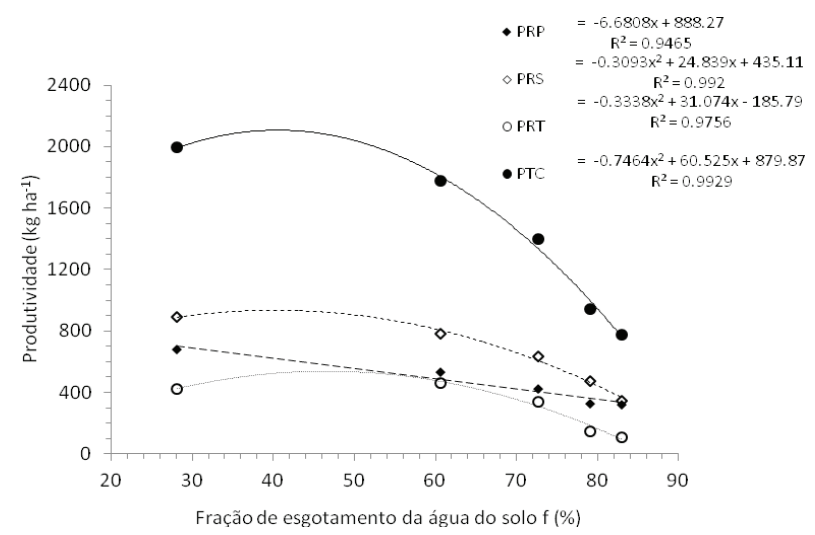

Figura 3. Produtividade de grãos de mamona, por ordem dos racemos primário $\left(\mathrm{PR}_{\mathrm{P}}\right)$, secundário $\left(\mathrm{PR}_{\mathrm{S}}\right)$, terciário $\left(\mathrm{PR}_{\mathrm{T}}\right)$ e total $\left(\mathrm{PT}_{\mathrm{C}}\right)$, em função da fração de esgotamento de água no solo (f) (Lavras, MG, 2008). cultura $\left(\mathrm{PT}_{\mathrm{C}}\right)$, verificou-se que o ponto de máxima produtividade $\left(2.095,46 \mathrm{~kg} \mathrm{ha}^{-1}\right)$ foi obtido com fração de esgotamento de água no solo de $41 \%$, o que corresponde a uma tensão de, aproximadamente, $20,8 \mathrm{kPa}$.

\section{CONCLUSÕES}

1. A eficiência de uso da água, para todos os racemos produzidos, reduziu-se, linearmente, com o aumento da restrição hídrica, sendo os maiores índices observados para o tratamento à menor tensão de $15 \mathrm{kPa}$, equivalente à fração de esgotamento de água do solo de $28,1 \%$.

2. Individualmente, em todos os tratamentos, a maior eficiência de uso da água e produtividade foram observadas nos racemos secundários.

3. A maior produtividade foi de $1.994,14 \mathrm{~kg} \mathrm{ha}^{-1}$, para a tensão de $15 \mathrm{kPa}$ e fração de esgotamento de água no solo de $28 \%$, e a menor foi de $773,91 \mathrm{~kg} \mathrm{ha}^{-1}$, para a tensão de $75 \mathrm{kPa}$ e fração de esgotamento de água no solo de $83 \%$.

\section{REFERÊNCIAS}

ALLEN, R. G. et al. Crop evapotranspiration: guidelines for computing crop water requirements. Rome: FAO, 1998. (Irrigation and drainage paper, 56).

BARROS JÚNIOR, G. et al. Consumo de água e eficiência do uso para duas cultivares de mamona submetidas a estresse hídrico. Revista Brasileira de Engenharia Agricola e Ambiental, Campina Grande, v. 12, n. 4, p. 350355, 2008.

BISCARO, G. A. et al. Produtividade de duas cultivares de mamona submetidas a diferentes lâminas de irrigação suplementar. Revista Brasileira de Engenharia Agrícola e Ambiental, Campina Grande, v. 16, n. 9, p. 925-930, 2012.

COMPANHIA NACIONAL DE ABASTECIMENTO (Conab). Acompanhamento da safra brasileira: grãos 2013/14. 2014. Disponível em: < http://www.conab.gov. br/OlalaCMS/uploads/arquivos/14_06_10_12_12_37 boletim_graos_junho_2014.pdf $>$. Acesso em: 5 jul. 2014.

COSTA, F. X. Disponibilidade de nutrientes no solo em função de doses de matéria orgânica no plantio da mamona. Revista Verde de Agroecologia e Desenvolvimento Sustentável, Mossoró, v. 5, n. 3, p. 204-212, 2010.

DANTAS, A. A. A.; CARVALHO, L. G.; FERREIRA, E. Classificação e tendência climática em Lavras, MG. Ciência e Agrotecnologia, Lavras, v. 31, n. 6, p. $1862-$ 1866, 2007. 
DOURADO NETO, D. et al. Software to model soil water retention curves (SWRC, version 2.0). Scientia Agricola, Piracicaba, v. 57, n. 1, p. 191-192, 2000.

DUARTE, J. M. de L. et al. Eficiência de uso da água na produção de óleo de girassol (Helliantus annuus L.) sob suspensão hídrica. Revista Brasileira de Agricultura Irrigada, Fortaleza, v. 6, n. 3, p. 166-175, 2012.

EMPRESA BRASILEIRA DE PESQUISA AGROPECUÁRIA (Embrapa). Centro Nacional de Pesquisa de Solos. Sistema brasileiro de classificação de solos. 2. ed. Rio de Janeiro: Embrapa, 2006.

FERREIRA, D. F. Sisvar: a computer statistical analysis system. Ciência e Agrotecnologia, Lavras, v. 35, n. 6, p. 1039-1042, 2011.

FREITAS, C. A. S. D. et al. Comportamento de cultivares de mamona em níveis de irrigação por gotejamento em Pentecoste, CE. Revista Brasileira de Engenharia Agrícola e Ambiental, Campina Grande, v. 14, n. 10, p. 1059-1066, 2010.

KOUTROUBAS, S. D.; PAPAKOSTA, D. K.; DOITSINIS, A. Adaptation and yielding ability of castor plant (Ricinus communis L.) genotypes in a Mediterranean climate. European Journal of Agronomy, Copenhague, v. 11, n. 3-4, p. 227-237, 1999.

LACERDA, R. D. de; GUERRA, H. O. C.; B. JÚNIOR, G. Influência do déficit hídrico e da matéria orgânica do solo no crescimento e desenvolvimento da mamoneira BRS 188 - Paraguaçu. Revista Brasileira de Ciências Agrárias, Recife, v. 4, n. 4, p. 440-448, 2009.

RODRIGUES, L. N. et al. Crescimento e produção de bagas da mamoneira irrigada com água residuária doméstica. Revista Brasileira de Engenharia Agrícola e Ambiental, Campina Grande, v. 13, supl., p. 825-835, 2009.
SAUSEN, T. L.; ROSA, L. M. G. Growth and carbon assimilation limitations in Ricinus communis (Euphorbiaceae) under soil water stress conditions. Acta Botanica Brasilica, Brasília, DF, v. 24, n. 3, p. 648-654, 2010.

SAVY FILHO, A. et al. Novo cultivar de mamona: IAC226 (Tabary). Bragantia, Campinas, v. 49, n. 2, p. 269280, 1990.

SILVA, L. C. da; BELTRÃO, N. E. de M. Incremento de fitomassa e produtividade do amendoinzeiro em função de lâminas e intervalos de irrigação. Revista Brasileira de Oleaginosas e Fibrosas, Campina Grande, v. 4, n. 2, p. 111-121, 2000.

SILVA, S. M. S. et al. Dotações hídricas em densidades de plantas na cultura da mamoneira cv. BRS Energia. Revista Brasileira de Ciências Agrárias, Recife, v. 4, n. 3, p. 338-348, 2009.

SOUZA, A. dos S. et al. Épocas de plantio e manejo da irrigação para a mamoneira: I. Componentes de produção. Revista Ciência Agronômica, Fortaleza, v. 38, n. 4, p. 414421, 2007a.

SOUZA, A. dos S. et al. Épocas de plantio e manejo da irrigação para a mamoneira: II. Crescimento e produtividade. Revista Ciência Agronômica, Fortaleza, v. 38, n. 4, p. 422-429, 2007 b.

VERÍSSIMO, M. A. A. et al. Rendimento de grãos de genótipos de mamona, semeados em três épocas, no Planalto Catarinense. Revista de Ciências Agroveterinárias, Lages, v. 8, n. 2, p. 129-138, 2009.

VIJAYA KUMAR, P. V. et al. Influence of moisture, thermal and photoperiodic regimes on the productivity of castor beans (Ricinus communis L.). Agricultural and Forest Meteorology, Hyderabad, v. 88, n. 1-4, p. 279-289, 1997. 